\title{
RECENT PERIODICALS RELATING TO THEOLOGICAL STUDIES
}

(I) ENGLish.

Church Quarterly Review, April 1901 (Vol. lii, No. 103: Spottiswoode \& Co.). Queen Victoria-Dr. Bright and Church HistoryHarnack's What is Christianity?-China of To-day-Canon Gore on the Eucharist - The Episcopate of Bishop Creighton-Priesthood and Sacrifice-Latin Versions of the Old Testament-Susan FerrierFrazer's 'Golden Bough'-A Practical Aspect of the Papacy-Dr. Moberly on the Atonement-Charles Henry Pearson-In Memoriam, Christopher Knight Watson-Short Notices.

Jewish Quarterly Review, April rgor (Vol. xiii, No. 51 : Macmillan \& Co.). S. Schechter Geniza specimens-G. B. Gray The 'Encyclopaedia Biblica' (vols. $i$ and ii) and the Textual Tradition of Hebrew proper names-E. N. Adler Auto de Fé and Jew-A. S. IsAacs The Talmud in History-M. STEINSCHNEIDER An Introduction to the Arabic Literature of the Jews (continued)-G. Margoliouth A Muhammadan Commentary on Maimonides' Misbneh Torah-I. AbraHamS Niese on the two Books of the Maccabees-D. Kaufmann Die Vertreibung der Marranen aus Venedig im Jahre 1550: Die Verbrennung der Talmudischen Litteratur in der Republik Venedig-D.S. Margoliouth The Legend of the Apostasy of Maimonides-W.BACHER $\mathrm{Zu}$ der von Algâhiz citirten Uebersetzung aus Jesaja-F. C. Conybeare Note on Weinstein's 'Zur Genesis der Agada'-M. Simon Josephus, Wars, v. 5, 7-E. N. Adler Karaitica-E. N. AdLer and I. Broyde An ancient Bookseller's Catalogue.

The Expositor, April Igor (Sixth Series, No. I6: Hodder \& Stoughton). F. Rendall The first Galatian ministry-T. K. Cheyne Few Things needful-J. H. Moulton Notes from the Papyri-J. DenneY The Doctrine of Sin-G. Matheson The Divine Will in NatureJ. A. Beet Immortality in Modern Theology-W. H. Bennetr Some recent Old Testament and other literature. 
May Igor (Sixth Series, No. I7). W. Sanday 'Atonement and Personality'-W. M. Ramsay The Apostolate; 'Am I not free?' Privileges of an Apostle; St. Peter in Corinth; Note on the Date of Second John; Had Paul seen Jesus?-G. Matheson Optimism and Pessimism - A. Carr Baptism for the Dead-J. A. BeET Immortality in Modern Theology-D. SMITH Had our Lord read the 'Tablet of Kebes'?-F. Fiewd Notes on Select Passages in the Old Testament.

June rgor (Sixth Series, No. I8). D. Smite Recent New Testament Criticism: (I) Its Methods-E. C. Selwyn The Christian Prophets at Philippi-G. Matheson Is Nature Morally Indifferent ?J. Denney The Gospel a Divine Righteousness-J. A. Beet The Eternal Punishment of Sin-J. Moffat The Autonomy of Jesus: a study in the Fourth Gospel.

\section{(2) American.}

The Presbyterian and Reformed Review, April Igor (Vol. xii, No. 46: MacCalla \& Co., Philadelphia). A. F. West Roman Autobiography and Augustine's Confessions-J. Lindsay Man's place in the Cosmos -A. T. Robertson Jesus and Questions of His Time-N. M. Stefrens Calvinism and the Theological Crisis-B. B. Warfield The Making of the Westminster Confessions-W. L LEDwith The Sermon as addressed to the Imagination-D. MOORE Mr. Wright and the Gospel of Luke-H. C. Minton Prof. Ormond's Foundations of Knowledge-Recent Theological Literature.

The American Joumal of Theology, April rgor (Vol. $\nabla$, No. 2: Chicago University Press). G. T. LadD Present religious tendencies in India-J. W. Ruchard The beginnings of Protestant worship in Germany and Switzerland-G. R. BERRY The Old Testament teaching concerning God-E. C. Bramhall The origin of the temporal privileges of Crusaders-The Hastings Bible Dictionary, vol. iii-Critical Notes : J. M. Whiton Some Implicates of Theism: F. D. Denis The Servant of Jehovah-Recent Theological Literature.

\section{(3) French and Belgian.}

Revue Biblique, April rgor (Vol. $x$, No. 2 : Paris, V. Lecoffre, for the School of the Convent of St. Stephen at Jerusalem). I. Guidi Il canone biblico della chiesa copta-A. van HOONACKER Notes sur l'histoire de la Restauration juive après l'exil de Babylone-L. Hackspill Etudes sur le milieu religieux et intellectuel contemporain du Nouveau Testament-M.-J. Lagrange Études sur les religions sémitiques : (2) Enceintes et pierres sacrées-Mélanges: P. Batiffol Les canons d'Hippolyte d'après des travaux récents: H. Grimme Mètres et 
strophes dans les fragments hébreux du manuscrit A de l'Ecclésiastique: A. Dombrovskr La doctrine de l'Église rasse et le canon de l'Ancien Testament-Chronique: H. VINcENT Monuments en pierres brutes dans la Palestine occidentale-Recensions-Bulletin.

Reoue de TOrient chriticn, IgoI (Vol. vi, No. I: Paris, A. Picard). $\mathrm{X}$. Griefs de l'Hellénisme contre la Russie-H. Lammens Les Nosairis furent-ils Chrétiens? A propos d'un livre récent-I. Guidr Vie et Récits de l'Abbé Daniel de Scété; (3) Texte copte (fin), (4) Corrections du texte éthiopien: Introduction par L. ClugneT-A. Palmierr L'ancienne. et Ia nouvelle théologie russe-F. NAU Lettre de Jacques d'Êdesse au diacre George-D. M. Girard Une découverte! Succession apostolique des Patriarches d'Arménie-Mélanges-Bibliographie.

Reoue d'histoire et de litterature religieuses, March-April 1901 (Vol. vi, No. 2: Paris, 74 Boulevard Saint-Germain). F. Cumont Le Taurobole et le culte de Bellone-A. Lorsy Les Mythes babyloniens et les premiers chapitres de la Genèse: (I) La Création, le chaos primordial; (2) Le combat du Créateur contre le Chaos-H. Cochin Le frère de Pétrarque et le livre du Repos des religieux, II-C MicheL Religions des peuples elassiques avant le christianisme: (3). Mythologie grecque-G. Mrssin Littérature religieuse moderne.

Analecta Bollandiana, March Igor (Vol. xx, No. I : Brussels, 14 Rue des Ursulines). Carmina de S. Quintino - J. Boyens Catalogus cadicum hagiographicorum graecorum bibliothecae monasterii Deiparae in Chalce insula-Miraculum S. Bernardi auctore Herberto-Bulletin des publications hagiographiques-U. ChevalreR Supplementurm ad Repertorium Hymnologicum (Deus altissime-Exultemus in Domino).

Reoue d'histoire ecclessiastique, April rgor (Vol. ii, No. 2 : Louvain, C. Peeters, for the Universite Catholique de Louvain). J. Flamion Les anciennes listes épiscopales des quatre grands siéges (continued)G. Vorsin La doctrine trinitaire đApollinaire de Laodicée (concluded) -U. BerLtèrRe Les origines de Citeaux et l'ordre bénédictin au xiïsiècle (concluded)-Mélanges : E. VAN RoEy La collection des 'Texte und Untersuchungen zur Geschichte der altchristlichen Literatur' (continued) - Comptes rendus-Chronique-Bibliographie.

\section{(4) GrRman.}

Theologische Quartalsthrift, 1901 (Vol. Lxxxiii, No. 2: Ravensburg and Vienna, H. Kitz). BRULL Die Ergreifung und Ueberlieferung Jesu an Pilatus-VeTter Die Zeugnisse der vorexilischen Propheten über den Pentateuch: (a) Hoseas (continwed and concluded)-Peters Textkritisches zu Hiob-FAULHABER Eine wertvolle Oxforder Handschrift 


\section{PERIODICALS RELATING TO THEOLOGICAL STUDIES $6_{39}$}

- Lederer Die Lehre des hl. Thomas von Aquin über den eigentlichen Beweggrund des Glaubens-Funk Die Berufung der allgemeinen Synoden des Altertums-Reviews - Analecta.

Neue kirchliche Zeitschrift, February 1901 (Vol. xii, No. 2 : Erlangen and Leipzig, A. Deichert). KLostermann Der 1 19: Psalm ein Gebet des Apostels Paulus: eine Neujahrsepistel an Herm D. R. Seeberg in Berlin-Cordes Die philosophische Erkenntnistheorie A. Ritschls - Notн Schleiermachers Monologen, II-CouARD Jüdische Sagen über das Leben Jesu.

March Igor (Vol xii, No. 3). Rabus Der Wesensunterschied von Protestantismus und Katholizismow-ZaHiN Katholische KritikTschackert Pasquilli de concilio Mantuanó indicium-Noth Schleiermachers Monologen (concluded)-CoUaRD Altchristliche Sagen über das Leben Jesu.

April rgor (Vol. xii, No. 4). F. LuNDGREEN Die Heilsbedeutung des Todes Jesu-M. Romperg Jerusalem und Galiläa, die Schauplätze der Erscheinungen des Auferstandenen-RABus Der Wesensunterschied von Protestantismus und Katholizismus (concluded).

May rgor (Vol. xii, No. 5). 'Schultzen Zur Lehre voń der Bekehrung-E. König Zur Erklärung und'Geschichte des DekalogsW. Schmidt Ethische Fragen: (6) Graf Leo Nikolajewitch Tolstoi-W. Vollert Christian August Anton Vollert.

Zeitschrift fïr Theologie und Kirche, May Igor (Vol. xi, Nos. 2 and 3 : Tübingen and Leipzig, J. C. B. Mohr). Gotrschick Augustins Anschauung von den Erlöserwirkungen Christi-Günther Arthur Bonus' Schriften-STuckert Sittliches Gesetz oder sittliches Gut ?Ritschl Religion und Sittlichkeit : ein Vortrag - GoTrschick Nachträge.

Theologische Studien und Kritiken, rgor (No. 3: Gotha, F. A. Perthes). Brederer Bemerkungen über die Art der Übersetzung im Targum Onkelos-Plath Zum Buch Tobit-Zimmermann Die vier ersten christlichen Schriften der Jerusalemischen Urgemeinde in den Synoptikern und der Apostelgeschichte-Mix Luther und Melanchthon in ihrer gegenseitigen Beurteilung-Notes : BARGE Uber eine vergessene Schrift Karlstadts-Reviews : Hering Kehrbach's Monumenta Germaniae Paedagogica.

Zeitschrift für Kirchengreschichte, March Igor (Vol.xxii, No. I: Gotha, F. A. Perthes). ERBes Petrus nicht in Rom, sondern in Jerusalem gestorben-Bess Die Annatenverhandlung der 'natio gallicana' des Konstanzer Konzils-Köhler Lutherana-Ribbeck Die Herzoge von Brieg und die geistlichen Patronatsherren, I-Brosch Die Ermordung der Veltliner Protestanten im Jahre 1620-Analecta: Barge Neue 


\section{THE JOURNAL OF THEOLOGICAL STUDIES}

Aktenstücke zur Geschichte der Wittenberger Unruhen von I521-22 : Clemen Zwei Gutachten Franz Lamberts von Avignon : Köster Beiträge zur Reformationsgeschichte Naumburgs von ${ }_{525}$ bis 1545 , I : GoETz Ein ungedruckter Brief Calvins.

Zeitschrift für die neutestamentliche Wissenschaft und die Kunde des Urchristentums, May rgor (Vol. ii, No. 2 : Giessen, J. Ricker). Th. Mommsen Die Rechtsverhältnisse des Apostels Paulus-H. HoltzMaNn Zum zweiten Thessalonicherbrief-C. Clemen Die Zahl des Tieres, Apoc. xiii I8-M. Steffen Das Verbältniss von Geist und Glauben bei Paulus, I-W. Soltau Zum Problem des Johannesevangeliums-Miscellanea: S. A. Fries Was meint Paulus mit 'Apaßia, Gal. i I 7 : E. Nestle I. Zur Didascalia Apostolorum, 2. Zum Vaterunser, 3. Luc. iv 18 , 19-Bibliography. 